VOL. $3(1970), 349-352$.

\title{
Endomorphism rings of non-abelian groups
}

\section{B. C. McQuarrie and J. J. Malone}

In 1942 F.W. Levi described those groups in which any two inner automorphic images of an arbitrary element commute. Until recently it was not known whether there existed non-abelian groups with the property that any two endomorphic images of an arbitrary element commute. Now, R. Faudree has given examples of finite $p$-groups having this property. In this paper we give a necessary and sufficient condition that a torsion group which contains no elements of order 2 has this property. The technique of proof involves looking at certain near rings of functions on the group. The inspiration for the theorem comes from the "halving" technique used by B.H. Neumann in 1940.

For $G$ an additively written group, let $T_{0}(G)$ denote the near ring of all functions $\alpha$ on $G$ to $G$ such that $O_{\alpha}=0$. Here, as in all our work with functions, addition of functions is done in a pointwise manner and multiplication of functions is done by composition. $E(G)(A(G), I(G))$ is the distributively generated (d.g.) near ring generated additively by the endomorphisms (automorphisms, inner automorphisms) of $G$. A. Fröhlich [3] gives much information about $\mathrm{d} \cdot \mathrm{g}$. near rings and was the first to remark that a d.g. near ring whose additive group is abelian is a ring. Thus the condition that any two endomorphic (automorphic, inner automorphic) images of an arbitrary element of $G$ commute is equivalent to the condition that $E(G)(A(G), I(G))$ is a ring. A group $G$ such that $E(G)(A(G), I(G))$ is a ring will be referred to as an $E$-group (A-group, I-group).

Received 10 August, 1970. 
DEFINITION. Let $l$ designate the identity map on $G$. If there exists in $T_{0}(G)$ a function $\lambda$ such that $\lambda+\lambda=1$, we say that $G$ has a halvable identity map.

Note that if $\lambda$ is a half of $l$ and $\alpha \in T_{0}(G)$, then $\alpha \lambda+\alpha \lambda=\alpha$

THEOREM 1. Let $G$ be a group with a halvable identity map. If $\lambda$ is a half of 1 then $E(G)(A(G), I(G))$ is a ming if $(\alpha+\beta) \lambda-\beta \lambda-\alpha \lambda=0$ for any $\alpha, \beta \in E(G)(A(G), I(G))$.

Proof. Assume that $\lambda$ distributes from the right over the sum of any pair of elements of $E(G)$. Then, for $\alpha$ and $\beta \in E(G)$,

$$
(\alpha+\beta) \lambda+(\alpha+\beta) \lambda=\alpha+\beta=\alpha \lambda+\alpha \lambda+\beta \lambda+\beta \lambda
$$

or

$$
\alpha \lambda+\beta \lambda+\alpha \lambda+\beta \lambda=\alpha \lambda+\alpha \lambda+\beta \lambda+\beta \lambda .
$$

Thus $\beta \lambda+\alpha \lambda=\alpha \lambda+\beta \lambda$. From this it follows that

$\alpha+\beta=(\alpha+\beta) \lambda+(\alpha+\beta) \lambda=\alpha \lambda+\beta \lambda+\alpha \lambda+\beta \lambda=\beta \lambda+\beta \lambda+\alpha \lambda+\alpha \lambda=\beta+\alpha$.

So $E(G)$ is a d.g. near ring whose additive group is abelian and $E(G)$ is, in fact, a ring.

It is immediate that a group which is of finite exponent and which contains an element of order 2 does not have a halvable identity map. On the other hand, the 2-quasicyclic group does have a halvable identity map. Among torsion-free groups the group of integers does not have a halvable identity map whereas the group of rationals does. If $G$ is a group of odd exponent $k$ then $\lambda$ may be taken as $\frac{k+1}{2} l$. (In the proof of Theorem 2 it is shown that $\frac{k+l}{2}$, is the unique half of 1.$)$ Thus $\lambda \in I(G)$ and the converse to Theorem 1 holds in this setting.

THEOREM 2. Let $G$ be a torsion group which contains no element of order 2 . Then the identity map on $G$ is uniquely halvable in $T_{0}(G)$. AZso, $(\alpha+\beta) \lambda-\beta \lambda-\alpha \lambda=0$ for any $\alpha, \beta \in E(G)(A(G), I(G))$ if and only if $E(G)(A(G), I(G))$ is a ring.

Proof. Define $\lambda$ in the following manner. For $g \in G$ such that 
$|g|=t$, set $g \lambda=\frac{t+1}{2} g$. Obviously, $\lambda+\lambda=1$. If $\mathrm{l}$ has another half, then for some $g \in G$ there exists $h \in G$ such that $h+h=g$ with $h \neq \frac{t+1}{2} g$. Let $|h|=s$. Then $s h=0$ or $h=(s-1)(-h)=\frac{s-1}{2}(-g)$. Thus $h \in\langle g\rangle$, the cyclic subgroup generated by $g$. But $g$ has only one half in $\langle g\rangle$. Thus $\lambda$ is the unique half of $l$ since, for an arbitrary $g \in G$, the equation $x+x=g$ has a unique solution in $G$.

If $\lambda$ is right distributive over $E(G)$, then Theorem $I$ assures us that $E(G)$ is a ring. Let us then assume that $E(G)$ is a ring and show that $\lambda$ is right distributive over $E(G)$.

Let $g \in G ; \alpha, \beta \in E(G)$. Note that $|g \alpha+g \beta|=1 . c \cdot m .\{|g \alpha|,|g \beta|\}$ and call this integer $t$. Then

$$
\begin{aligned}
g((\alpha+\beta) \lambda)=(g \alpha+g \beta) \lambda=\frac{t+1}{2}(g \alpha+g \beta)=\frac{t+1}{2} g \alpha+\frac{t+1}{2} g \beta \\
\quad=(g \alpha) \lambda+(g \beta) \lambda=g(\alpha \lambda+\beta \lambda)
\end{aligned}
$$

as desired.

Note that if the $G$ of Theorem 2 is not of finite exponent, it is not assumed that $\lambda \in E(G)$.

THEOREM 3. Let $G$ be a torsion group which contains no element of order 2 . Then $\lambda$ is right distributive over $E(G)(A(G), I(G))$ if and only if $\lambda \alpha=\alpha \lambda$ for each $\alpha \in E(G)(A(G), I(G))$.

Proof. If $\lambda$ is in the (multiplicative) centralizer of $E(G)$ in $T_{0}(G)$, then obviously $\lambda$ is right distributive over $E(G)$.

Assume that $\lambda$ is right distributive over $E(G)$ and let $\alpha \in E(G)$. Now $\alpha$ can be written as a finite sum of endomorphisms and/or anti-endomorphisms of $G$, say $\alpha=\alpha_{1}+\ldots+\alpha_{n}$. Let $t=1 . \operatorname{cm} \cdot\left\{|g|,\left|g \alpha_{1}\right|, \ldots,\left|g \alpha_{n}\right|\right\}$. First we see that $g\left(\alpha_{i} \lambda\right)=\frac{t+1}{2}\left(g \alpha_{i}\right)=\left(\frac{t+1}{2} g\right) \alpha_{i}=g\left(\lambda \alpha_{i}\right)$. Then the theorem follows since 


$$
\begin{aligned}
g(\alpha \lambda)=g\left(\left(\alpha_{1}+\ldots+\alpha_{n}\right) \lambda\right)=g\left(\alpha_{1} \lambda+\ldots+\alpha_{n} \lambda\right)=g\left(\lambda \alpha_{1}+\ldots+\right. & \left.\lambda \alpha_{n}\right) \\
& =g(\lambda \alpha) .
\end{aligned}
$$

A.J. Chandy [1] has applied the work of F.W. Levi [4] to the study of $I(G)$. A group is an $I$-group if and only if the commutator of any two elements of the group commutes with each of the two elements. An I-group is nilpotent of class at most 3 ; an I-group which does not contain an element of order 3 is nilpotent of class at most 2 . There exists no general theory of A-groups or of E-groups. The first examples of non-abelian $E$-groups are given in [2].

\section{References}

[1] A.J. Chandy, "Rings generated by inner automorphisms of non-abelian groups", Doctoral thesis, Boston University, 1965.

[2] R.J. Faudree, "Groups in which each element commutes with its endomorphic images", Proc. Amer. Math. Soc. (to appear).

[3] A. Fröhlich, "Distributively generated near-rings", Proc. London Math. Soc. (3) 8 (1958), 76-108.

[4] F.W. Levi, "Groups in which the commutator operation satisfies certain algebraic conditions", J. Indian Math. Soc. (N.S.) 6 (1942), $87-97$.

[5] B.H. Neumann, "On the commutativity of addition", J. London Math. Soc. 15 (1940), 203-208.

Worcester Polytechnic Institute, Worcester, Massachusetts, USA, and Texas A \& M University, College Station, Texas, USA. 